\title{
A critical appraisal of the clinical effectiveness of a fixed combination of valsartan, amlodipine, and hydrochlorothiazide in achieving blood pressure goals
}

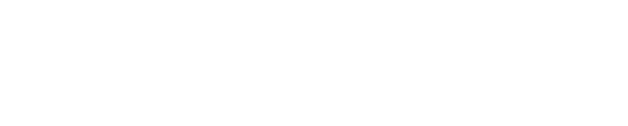

\author{
Cheryl L Laffer' \\ Fernando Elijovich ${ }^{2}$ \\ 'Section of Hypertension and Vascular \\ Medicine, ${ }^{2}$ Division of General \\ Internal Medicine, Department of \\ Medicine, Texas A\&M Health Sciences \\ Center College of Medicine, Temple, \\ TX USA
}

\begin{abstract}
Recent guidelines for the treatment of hypertension have focused on the need for multiple medications to get most patients to goal blood pressure (BP). Two to three different classes of antihypertensive agents are frequently required, increasing the risk of poor compliance with therapy. Hence, the guidelines have recommended starting with combination therapy in patients with BP that is over $20 \mathrm{~mm} \mathrm{Hg}$ systolic or $10 \mathrm{~mm} \mathrm{Hg}$ diastolic above goal. The latest advance in treatment regimen has been the development of triple-therapy combinations of an angiotensin receptor blocker, amlodipine, and hydrochlorothiazide. We review the pathophysiologic rationale for such a combination and the efficacy, safety, and tolerability of the first triple therapy that has become available: valsartan + amlodipine + hydrochlorothiazide. Finally, we suggest that use of triple therapy could improve the accuracy of diagnosing resistant hypertension, an increasingly prevalent and severe condition, by enhancing adherence to treatment and weeding out patients with pseudoresistance. This would allow for implementation of expensive and invasive workup only in those truly resistant patients in whom it is justified.
\end{abstract}

Keywords: combination therapy, compliance, hypertension control rates, resistant hypertension

Hypertension remains the most prevalent chronic disease in the developed world, and the number one reason for a patient to seek medical care. In addition, it is a major contributor to cardiovascular and renal morbidities and mortalities. Epidemiologic studies have demonstrated that even very small changes in population blood pressure (BP) (2 mm Hg) could have a major impact on ischemic heart disease or stroke mortality (7\% and 10\% decreases, respectively). ${ }^{1}$ Despite this recognition, control rates of hypertension continue to lag behind national goals.

The latest US data from the National Health and Nutrition Examination Survey ${ }^{2}$ have shown a dramatic improvement in control of hypertension in the US over the last 10 years, with $50 \%$ of hypertensive subjects reaching goal BP of less than 140/90 mm Hg. Unfortunately, this also means that 50\% remain uncontrolled, and only $72 \%$ of all hypertensive subjects are treated. Reasons for poor control rates are multifactorial, including societal (eg, patient lack of understanding, compliance, or access to care or medications), physician inertia (failure to initiate or increase therapy for uncontrolled hypertension), and complications of treatment (side effects, inconvenience of multiple doses, interfering substances). The use of combination therapy has helped to reduce the impact of many of these factors, and the Seventh 
Report of the Joint National Committee on Prevention, Detection, Evaluation, and Treatment of High Blood Pressure $(\text { JNC7, 2003) })^{3}$ was the first guideline to specifically advocate their use for these purposes. For subjects whose BP was at least 20/10 $\mathrm{mm} \mathrm{Hg}$ above goal, the JNC7 recommended starting with a two-drug combination. The European guidelines of 2007 followed suit, also recommending combination treatment for those with elevated cardiovascular risk. ${ }^{4}$ Not only is such treatment likely to improve the rate at which BP goal is achieved ${ }^{5,6}$ but also it has been shown that more rapid control of BP improves adherence by enhancing trust in the physician-patient relationship.,

A further argument to support the use of combination therapy is the number of medications required to achieve goal BP in most patients. Publication of multiple large outcome trials in patients with hypertension over the last decade (eg, LIFE [Losartan Intervention for Endpoint Reduction in Hypertension], ALLHAT [Antihypertensive and Lipid-lowering Treatment to Prevent Heart Attack Trial], and ASCOT-BPLA [Anglo-Scandinavian Cardiac Outcomes Trial - Blood Pressure Lowering Arm]) has shown that most hypertensive subjects will require at least two medications. ${ }^{8-10}$ Patients with increased cardiovascular or renal risk require 3.2 medications, on average, to achieve their lower goal BP. ${ }^{11}$ Recognition of this need has led to the marketing of multiple two-drug combinations, eg, thiazide diuretics with $\beta$-blockers, angiotensin-converting enzyme inhibitors (ACEIs), or angiotensin II type 1 receptor antagonists (ARBs); nondihydropyridine calcium channel blockers (CCBs) with ACEIs; and dihydropyridine (DHP) CCBs with ACEIs or ARBs. A number of these are approved by the US Food and Drug Administration (FDA) as first-line therapy for hypertension. The latest efforts have extended this approach to the development of three-drug combinations of ARB + DHPCCB + thiazide, eg, valsartan + amlodipine + hydrochlorothiazide. Although triple therapy with modern antihypertensive agents may seem to be an entirely new development, there is historical precedent for this approach. A product combining reserpine, hydralazine, and hydrochlorothiazide has been available for decades, and the combination of these agents was shown to be safe and effective in one of the earliest Veterans Administration trials. ${ }^{12}$

The choice of which two agents to combine in one pill has relied on our current understanding of the many pathophysiological mechanisms underlying essential hypertension. Probably the best known of these mechanisms is the interaction between the renin-angiotensin-aldosterone system (RAAS) and sodium balance in determining BP.
Studies carried out in the 1970s using the Goldblatt rat models of renovascular hypertension laid the foundation of our understanding of this interaction. In the two-kidney, one-clip (2K1C) model, renin secreted by the clipped kidney leads to renin-dependent hypertension. This is sustained long term via pressure natriuresis by the contralateral, nonclipped kidney, because preserved or reduced plasma volume maintains stimulation of continued renin secretion by the clipped kidney. ${ }^{13}$ In contrast, in the uninephrectomized, $1 \mathrm{~K} 1 \mathrm{C}$ model, initial stimulation of renin secretion is soon inhibited by volume expansion because of absent pressure natriuresis, leading to diuretic-sensitive, renin-independent hypertension. ${ }^{14}$ This is the rationale for combining agents that are effective in renin-dependent hypertension, such as $\beta$-blockers or RAAS-blockers, with those that are more effective in renin-independent forms, such as diuretics. Whereas the vasodilating CCBs are efficacious in both renin-dependent and renin-independent hypertension, they stimulate renin release by renal vasodilation, natriuresis, and the lowering of BP itself, an effect that can be counteracted by combining them with an RAAS-blocker. ${ }^{15}$ Two-drug combinations have been available for some time and have been shown to improve adherence, ${ }^{16}$ outcomes, ${ }^{10,17}$ and overall rates of adverse events, and to reduce BP more promptly, ${ }^{18}$ compared with their individual components.

Additional recent knowledge derived from clinical trials was the most likely determinant for the selection of the three agents combined in the first triple-therapy pill released in the US: valsartan + amlodipine+hydrochlorothiazide. The renin-dependent and -independent components of BP elevation are addressed by the ARB and the diuretic components of this combination, respectively, whereas the CCB amlodipine provides potent antihypertensive action for all subjects. Moreover, there is proof from clinical trials that the ARB valsartan is effective in the treatment of high-risk hypertension (VALUE [Valsartan Antihypertensive Long-term Use Evaluation] trial), ${ }^{19}$ congestive heart failure (Val-HeFT [Valsartan Heart Failure Trial]), ${ }^{20}$ and ischemic heart disease (VALIANT [Valsartan in Acute Myocardial Infarction Trial]). ${ }^{21}$ Furthermore, as with all ARBs, this agent exhibits adverse event rates that are essentially indistinguishable from placebo. Amlodipine was more effective than valsartan at reducing the secondary outcomes of myocardial infarction and stroke in VALUE, owing largely to more rapid lowering of BP. ${ }^{19}$ The concern of worsening heart failure with DHPCCBs due to reflex sympathetic activation by vasodilation was dispelled for amlodipine years ago in the PRAISE (Prospective Randomized Amlodipine Survival Evaluation) trial. ${ }^{22}$ Furthermore, the 
potential adverse effect of amlodipine on progression of renal disease via afferent arteriolar vasodilatation and glomerular hyperfiltration, as suggested in the AASK ${ }^{23}$ (African American Study of Kidney Disease and Hypertension) trial, should be attenuated by the concomitant efferent arteriolar dilatation by the RAAS-blocker. Finally, thiazide-induced stimulation of renin release potentiates the effect of the ARB in the combination, whereas, conversely, blockade of the proinflammatory and pro-oxidant effects of angiotensin II by the ARB contributes to attenuation of the potential adverse metabolic effects of this diuretic. ${ }^{24}$

The efficacy and safety of this triple combination were demonstrated by double-blind comparison of maximum usual doses of the three-drug regimen to each of the three possible two-drug combinations of its constituents in 4285 subjects with stage 2 hypertension. ${ }^{25}$ Following the initial 2-4 weeks of treatment on the lower dosages, patients were titrated to maximal doses of the dual or triple combinations. After 8 weeks, triple therapy produced significantly greater reductions in systolic (6.2-8.2 $\mathrm{mm} \mathrm{Hg}$ ) and diastolic (3.3-5.3 $\mathrm{mm} \mathrm{Hg}$ ) $\mathrm{BP}$ and greater rates of control (71\% vs 45\%-54\%) compared with each of the dual-combination therapies. These effects were independent of age, gender, race, ethnicity, or baseline $\mathrm{BP}^{25}$ In a secondary analysis, triple therapy was shown to reduce BP faster and with a greater effect in those with higher baseline systolic BP, without increasing adverse effects. ${ }^{26} \mathrm{~A}$ different design was employed in a trial of stage 2 hypertension in black patients. They were initially treated with amlodipine monotherapy or with the dual valsartan + amlodipine combination. Hydrochlorothiazide, if needed to achieve systolic BP $<130$, was added after 8 weeks. The resulting triple-therapy group sustained greater BP reduction compared with those who ultimately received amlodipine + hydrochlorothiazide dual therapy. ${ }^{27}$

The valsartan + amlodipine + hydrochlorothiazide tripletherapy combination is not FDA approved for first-line use in hypertension. It is indicated for use in patients who remain uncontrolled on any two of the component classes. This combination does not worsen either hypokalemia or hyperkalemia compared with the approved valsartan + hydrochlorothiazide combination. In addition, rates of edema are lower with the triple combination than with the approved valsartan + amlodipine dual therapy, ${ }^{25}$ most likely reflecting combined effects of postcapillary vasodilation by valsartan and volume contraction by hydrochlorothiazide. The only absolute contraindication to its use, as for any preparation containing an RAAS-blocker, is pregnancy. There is no evidence of the need for dose adjustment in the elderly.
There have been no published comparisons of this triple therapy with a similar regimen employing a different ARB. However, a comparison of treatment with valsartan + amlodipine + hydrochlorothiazide with losartan + hydrochlorothiazide treatment in patients with stage 2 hypertension was presented at the American Society of Hypertension meeting in May 2010. In this study, the triple therapy showed faster and greater BP reduction than did the losartan-based dual therapy. In addition, subjects who were initially randomized to dual-therapy arms and subsequently switched to triple therapy sustained the expected additional decrease in BP and increase in control rates. ${ }^{28}$

Results of treatment with another triple-therapy combination using the ARB olmesartan with amlodipine and hydrochlorothiazide were recently published. ${ }^{29}$ In this study, 2492 patients with stage 2 hypertension were initially randomized to one of the three possible highest-dose dual combinations for 2-4 weeks. Half of them were later switched to the highest-dose triple-therapy combination for a further 8 weeks. Triple therapy produced an additional 7.1-9.6 $\mathrm{mm} \mathrm{Hg}$ reduction in systolic BP and a 3.8-6.7 $\mathrm{mm} \mathrm{Hg}$ reduction in diastolic BP compared with dual therapy, with increased control rates (70\% vs 41\%-53\%) and without an increase in adverse effects. ${ }^{29}$

In summary, triple combinations of an ARB with amlodipine and hydrochlorothiazide have been shown to be safe and effective in lowering BP in moderate to severe hypertension.

We have suggested that there may be a role for triple therapy in making an accurate diagnosis of resistant hypertension, ${ }^{30}$ which is a major problem that is increasing in prevalence. Resistant hypertension is defined as the inability to achieve risk factor-adjusted goal BP (less than 140/90 in uncomplicated hypertensive patients, and less than 130/80 in those with diabetes or confirmed cardiovascular or chronic kidney disease), despite optimal doses of three agents from different families, one of which is a diuretic. ${ }^{31}$ Diuretics not only potentiate the action of most other antihypertensive therapies but also are uniquely efficacious in the $50 \%$ of hypertensive patients with salt-sensitive BP. ${ }^{31,32}$ These subjects are at increased cardiovascular risk, independent of the severity of their hypertension, ${ }^{33}$ via mechanisms that are not yet completely understood. ${ }^{34,35}$ The current prevalence of resistant hypertension is estimated at $20 \%-30 \%$ of all hypertensive patients. It is more common in certain subgroups (eg, elderly, black, obese, diabetic, and those with hypertensive heart disease and chronic kidney disease) $)^{31}$ who have a higher prevalence of salt sensitivity of BP, and is likely to increase as the population ages and becomes more obese. 
Making an accurate diagnosis of resistant hypertension is of the utmost importance in order to avoid unnecessary, costly, and invasive workup for an overlooked cause (such as secondary hypertension) in subjects who may have pseudoresistance. Recognized causes for the latter include lack of compliance owing to complex medication regimens in patients with hypertension and multiple comorbidities, or discouragement by the lack of success of BP control with slow therapeutic regimens employing a stepwise approach to the addition of drugs or dose escalation. We have suggested that decreased pill burden and rapid escalation from two-drug regimens (in separate or combined pills) to a single-pill, triple-therapy approach may diminish pseudoresistance by increasing compliance and adherence ${ }^{36}$ with ensuing control of BP in these subjects. This would allow for quicker and more accurate identification of a smaller number of subjects with truly resistant hypertension, in whom an aggressive workup may be indicated appropriately, despite its risks and costs.

\section{Conclusions}

The combination valsartan + amlodipine + hydrochlorothiazide has been shown to be safe and effective in lowering BP across a broad spectrum of hypertensive populations (young and old, male and female, black and white) with moderate to severe hypertension. By combining agents with differing antihypertensive mechanisms, this therapy reduces side effects of the individual classes while enhancing their actions. Control rates are improved over dual therapy and are achieved more rapidly, thereby enhancing compliance. Our contention is that use of such a triple-therapy combination could lead to better recognition of truly resistant hypertension, which is becoming an increasingly prevalent problem as society ages and becomes more obese.

\section{Disclosure}

The authors report no conflicts of interest in this work.

\section{References}

1. Lewington S, Clarke R, Qizilbash N, et al. Age-specific relevance of usual blood pressure to vascular mortality: a meta-analysis of individual data for one million adults in 61 prospective studies. Lancet. 2002; 360:1903-1913.

2. Egan BM, Zhao Y, Axon RN. US trends in prevalence, awareness, treatment and control of hypertension, 1988-2008. JAMA. 2010; 303:2043-2050.

3. Chobanian AV, Bakris GL, Black HR, et al. Seventh report of the Joint National Committee on Prevention, Detection, Evaluation, and Treatment of High Blood Pressure. Hypertension. 2003;42:1206-1252.

4. Mancia G, De Backer G, Dominiczak A, et al. 2007 guidelines for the management of arterial hypertension: the Task Force for the Management of Arterial Hypertension of the European Society of Hypertension (ESH) and of the European Society of Cardiology (ESC). Eur Heart J. 2007;28:1462-1536.
5. Staessen JA, Thijisq L, Fagard R, et al. Effects of immediate versus delayed antihypertensive therapy on outcome in the Systolic Hypertension in Europe Trial. J Hypertens. 2004;22:847-857.

6. Weber MA, Julius S, Kjeldsen SE, et al. Blood pressure dependent and independent effects of antihypertensive treatment on clinical events in the VALUE trial. Lancet. 2004;363:2049-2051.

7. Gradman AH, Acevedo C. Evolving strategies for the use of combination therapy in hypertension. Curr Hypertens Rep. 2002;4:343-349.

8. Dahlof B, Devereux RB, Kjeldsen SE, et al. Cardiovascular morbidity and mortality in the Losartan Intervention for Endpoint Reduction in Hypertension study (LIFE): a randomised trial against atenolol. Lancet. 2002;359:995-1003.

9. The ALLHAT Officers and Coordinators for the ALLHAT Collaborative Research Group. Major outcomes in high-risk hypertensive patients randomized to angiotensin-converting enzyme inhibitor or calcium channel blocker vs diuretic: the Antihypertensive and Lipid-lowering Treatment to Prevent Heart Attack Trial (ALLHAT). JAMA. 2002; 288:2981-2997.

10. Dahlof B, Sever PS, Poulter NR, et al. Prevention of cardiovascular events with an antihypertensive regimen of amlodipine adding perindopril as required versus atenolol adding bendroflumethiazide as required, in the Anglo-Scandinavian Cardiac Outcomes Trial-Blood Pressure Lowering Arm (ASCOT-BPLA): a multicentre randomised controlled trial. Lancet. 2005;366:895-906.

11. Blood Pressure Lowering Treatment Trialists' Collaboration. Effects of different blood-pressure-lowering regimens on major cardiovascular events: results of prospectively-designed overviews of randomised trials. Lancet. 2003;362:1527-1535.

12. Veterans Administration Cooperative Study Group on Antihypertensive Patients. Effects of treatment on morbidity in hypertension: results in patients with diastolic blood pressures averaging 115 through 129 mm Hg. JAMA. 1967;202:1028-1034

13. Gavras H, Brunner HR, Thurston H, Laragh JH. Reciprocation of renin dependency with sodium volume dependency in renal hypertension. Science. 1975;188:1316-1317.

14. Gavras H, Brunner HB, Vaughan ED, Laragh JH. Angiotensin-sodium interaction in blood pressure maintenance of renal hypertensive and normotensive rats. Science. 1973;180:1369-1371.

15. Jamerson KA, Basile J. Prompt, aggressive BP lowering in high-risk patients. J Clin Hypertens (Greenwich). 2008;10:40-48.

16. Taylor AA, Shoheiber O. Adherence to antihypertensive therapy with fixed-dose amlodipine besylate/benazepril $\mathrm{HCl}$ versus comparable component-based therapy. Congest Heart Fail. 2003;9:324-332.

17. Jamerson K, Weber MA, Bakris GL, et al. Benazepril plus amlodipine or hydrochlorothiazide for hypertension in high-risk patients. $N$ Engl J Med. 2008;59:2417-2428.

18. Bakris GL, Weir MR. Achieving goal blood pressure in patients with type 2 diabetes: conventional versus fixed-dose combination approaches. J Clin Hypertens (Greenwich). 2003;5:202-209.

19. Julius S, Kjeldsen SE, Weber M, et al. Outcomes in hypertensive patients at high cardiovascular risk treated with regimens based on valsartan or amlodipine: the VALUE randomised trial. Lancet. 2004;363: 2022-2031.

20. Cohn JN, Tognoni G; for the Valsartan Heart Failure Trial Investigators. A randomized trial of the angiotensin-receptor blocker valsartan in chronic heart failure. $N$ Engl J Med. 2001;345:1667-1675.

21. Pfeffer MA, Mcmurray JJ, Velazquez EJ, et al. Valsartan, captopril, or both in myocardial infarction complicated by heart failure, left ventricular dysfunction, or both. N Engl J Med. 2003;349:1893-1906.

22. Packer M, O'Connor CM, Ghali JK, et al. Effect of amlodipine on morbidity and mortality in severe chronic heart failure. $\mathrm{N}$ Engl J Med. 1996;335:1107-1114.

23. Wright JT Jr, Bakris G, Greene T, et al. Effect of blood pressure lowering and antihypertensive drug class on progression of hypertensive kidney disease: results from the AASK trial. JAMA. 2002;288:2421-2431.

24. Motwani JG. Combining renin-angiotensin-aldosterone system blockade with diuretic therapy for treatment of hypertension. J Renin Angiotensin Aldosterone Syst. 2002;3:72-78. 
25. Calhoun DA, Lacourciere Y, Chiang YT, Glazer RD. Triple antihypertensive therapy with amlodipine, valsartan, and hydrochlorothiazide: a randomized clinical trial. Hypertension. 2009;54:32-39.

26. Calhoun DA, Crikelair NA, Yen J, Glazer RD. Amlodipine/valsartan/ hydrochlorothiazide triple combination therapy in moderate/severe hypertension: secondary analyses evaluating efficacy and safety. Adv Therapy. 2009;26:1012-1023.

27. Flack JM, Calhoun DA, Satlin L, et al. Efficacy and safety of initial combination therapy with amlodipine/valsartan compared with amlodipine monotherapy in black patients with stage 2 hypertension: the EX-STAND study. J Hum Hypertens. 2009;23:479-489.

28. Wright R, Duprez D, Yadao A, et al. A valsartan-based antihypertensive regimen is more effective than a losartan-based regimen in patients with stage 2 hypertension: the EXALT study [Abstract]. J Clin Hypertens. 2010;12:533

29. Oparil S, Melino M, Lee J, et al. Triple therapy with olmesartan medoxomil, amlodipine besylate, and hydrochlorothiazide in adult patients with hypertension: the TRINITY multicenter, randomized, double-blind, 12-week, parallel-group study. Clin Ther. 2010;32:1-18.

30. Elijovich F, Laffer C. A role for single-pill triple therapy in hypertension. Ther Adv Cardiovasc Dis. 2009;3:231-240.
31. Calhoun DA, Jones D, Textor S, et al. Resistant hypertension: diagnosis, evaluation, and treatment. A scientific statement from the American Heart Association Professional Education Committee of the Council for High Blood Pressure Research. Hypertension. 2008;51:1403-1419.

32. Laffer CL, Elijovich F. Essential hypertension of Caribbean Hispanics: sodium, renin, and response to therapy. J Clin Hypertens (Greenwich). 2002;4:266-273.

33. Weinberger MH, Fineberg NS, Fineberg SE, Weinberger M. Salt sensitivity, pulse pressure, and death in normal and hypertensive humans. Hypertension. 2001;37:429-432.

34. Laffer CL, Laniado-Schwartzman M, Wang MH, et al. Differential regulation of natriuresis by 20-hydroxyeicosatetraenoic acid in human salt-sensitive versus salt-resistant hypertension. Circulation. 2003; 107:574-578.

35. Laffer CL, Gainer JV, Waterman MR, et al. The T8590C polymorphism of CYP4A11 and 20-hydroxyeicosatetraenoic acid in essential hypertension. Hypertension. 2008;51:767-772.

36. Gerbino PP, Shoheiber O. Adherence patterns among patients treated with fixed-dose combination versus separate antihypertensive agents. Am J Health Syst Pharm. 2007;64:1279-1283.
Integrated Blood Pressure Control

\section{Publish your work in this journal}

Integrated Blood Pressure Control is an international, peer-reviewed open-access journal focusing on the integrated approach to managing hypertension and risk reduction. Treating the patient and comorbidities together with diet and lifestyle modification and optimizing healthcare resources through a multidisciplinary team approach constitute key

\section{Dovepress}

features of the journal. This journal is indexed on American Chemical Society's Chemical Abstracts Service (CAS). The manuscript management system is completely online and includes a very quick and fair peerreview system, which is all easy to use. Visit http://www.dovepress.com/ testimonials.php to read real quotes from published authors.

Submit your manuscript here: http://www.dovepress.com/integrated-blood-pressure-control-journal 